\title{
Existence and monotonicity of positive solutions for hybrid Caputo-Hadamard fractional integro-differential equations
}

\author{
Abdelouaheb Ardjouni*1 and Moussa Haoues ${ }^{2}$ \\ ${ }^{1}$ Department of Mathematics and Informatics, University of Souk Ahras, P.O. Box 1553, Souk Ahras, 41000, Algeria. \\ ${ }^{2}$ Laboratory of Informatics and Mathematics, Department of Mathematics and Informatics, University of Souk-Ahras, P.O. Box 1553,
}

Souk-Ahras, 41000, Algeria.

Received 08 August 2021; Accepted 27 September 2021

\begin{abstract}
The purpose of this paper is to present new results on the existence, uniqueness and monotonicity of positive solutions for hybrid Caputo-Hadamard fractional integro-differential equations. Our results are based on the method of upper and lower solutions, and the Dhage and Banach fixed point theorems. Two examples are given to illustrate our obtained results.
\end{abstract}

AMS Subject Classifications: 40A05, 40A99, 46A70, 46A99.

Keywords: Fractional integro-differential equations, fixed point theorems, existence, positivity, monotonicity.

\section{Contents}

1 Introduction

\section{Introduction}

Fractional differential equations with and without delay arise from a variety of applications including in various fields of science and engineering such as applied sciences, physics, chemistry, biology, medicine, etc. In particular, problems concerning qualitative analysis of fractional differential equations with and without delay have received the attention of many authors, see [1]-[14], [16]-[22] and the references therein.

Hybrid Fractional differential equations arise from a variety of different areas of applied mathematics and physics, e.g., in the deflection of a curved beam having a constant or varying cross section, a three-layer beam, electromagnetic waves or gravity driven flows and so on $[2,3,13,14,21,22]$.

\footnotetext{
*Corresponding author. Email address: abd_ardjouni@yahoo.fr (Abdelouaheb Ardjouni), moussakoussa84@yahoo.fr (Moussa
} Haoues) 
Hybrid Caputo-Hadamard fractional integro-differential equations

Let $J=\left[t_{0}, T\right]$. Haoues et al. [18] investigated the existence, uniqueness and monotonicity of positive solutions for the following hybrid fractional integro-differential equation

$$
\left\{\begin{array}{l}
{ }^{C} D_{t_{0}}^{\alpha}\left(\frac{x(t)}{p(t)+\frac{1}{\Gamma(\beta)} \int_{t_{0}}^{t}(t-s)^{\beta-1} g(s, x(s)) d s}\right)=f(t, x(t)), t \in J, \\
x\left(t_{0}\right)=p\left(t_{0}\right) \theta \geq 0,
\end{array}\right.
$$

where ${ }^{C} D_{t_{0}}^{\alpha}$ is the Caputo fractional derivative of order $0<\alpha \leq 1,0<\beta \leq 1,0 \leq t_{0}<T, f, g: J \times \mathbb{R} \rightarrow \mathbb{R}$ and $p: J \rightarrow \mathbb{R}$ are given continuous functions. By using the method of the upper and lower solutions and the Dhage and Banach fixed point theorems, the authors obtained the existence, uniqueness and monotonicity of a positive solution.

In this paper, we extend the results in [18] by proving the existence, uniqueness and monotonicity of positive solutions for the following hybrid nonlinear Caputo-Hadamard fractional integro-differential equation

$$
\left\{\begin{array}{l}
{ }^{C H} D_{t_{0}}^{\alpha}\left(\frac{x(t)}{p(t)+\frac{1}{\Gamma(\beta)} \int_{t_{0}}^{t}\left(\log \frac{t}{s}\right)^{\beta-1} g(s, x(s)) \frac{d s}{s}}\right)=f(t, x(t)), t \in J, \\
x\left(t_{0}\right)=p\left(t_{0}\right) \theta \geq 0,
\end{array}\right.
$$

where ${ }^{C H} D_{t_{0}}^{\alpha}$ is the Caputo-Hadamard fractional derivative of order $0<\alpha \leq 1,0<\beta \leq 1,1 \leq t_{0}<T$, $f, g: J \times \mathbb{R} \rightarrow \mathbb{R}$ and $p: J \rightarrow \mathbb{R}$ are given continuous functions. To prove the existence, uniqueness and monotonicity of positive solutions, we transform (1.1) into an integral equation and then by the method of upper and lower solutions and use Dhage and Banach fixed point theorems.

\section{Preliminaries}

Let $X=C(J)$ be the Banach space of all real-valued continuous functions defined on the compact interval $J$, endowed with the maximum norm. Define the subset $\mathcal{C}_{\theta}=\left\{x \in X: x(t) \geq p\left(t_{0}\right) \theta, t \in J\right\}$ of $X$.

Definition 2.1 ([19]). The Hadamard fractional integral of order $\alpha>0$ for a continuous function $x:\left[t_{0},+\infty\right) \rightarrow$ $\mathbb{R}$ is defined as

$$
{ }^{H} I_{t_{0}}^{\alpha} x(t)=\frac{1}{\Gamma(\alpha)} \int_{t_{0}}^{t}\left(\log \frac{t}{s}\right)^{\alpha-1} x(s) \frac{d s}{s} .
$$

Definition 2.2 ([19]). The Caputo-Hadamard fractional derivative of order $\alpha>0$ for a continuous function $x:\left[t_{0},+\infty\right) \rightarrow \mathbb{R}$ is defined as

$$
{ }^{C H} D_{t_{0}}^{\alpha} x(t)=\frac{1}{\Gamma(n-\alpha)} \int_{t_{0}}^{t}\left(\log \frac{t}{s}\right)^{n-\alpha-1} \delta^{n}(x)(s) \frac{d s}{s},
$$

where $\delta^{n}=\left(t \frac{d}{d t}\right)^{n}$ and $n=[\alpha]+1$.

Lemma 2.3 ([19]). Let $\alpha>0$ and $x \in C^{n-1}\left[t_{0},+\infty\right)$ and $\delta^{n}(x)$ exists almost everywhere on any bounded interval of $\left[t_{0},+\infty\right)$. Then

$$
\left({ }^{H} I_{t_{0}}^{\alpha C H} D_{t_{0}}^{\alpha} x\right)(t)=x(t)-\sum_{k=0}^{n-1} \frac{x^{(k)}\left(t_{0}\right)}{k !}(\log t)^{k} .
$$

In particular, when $0<\alpha \leq 1,\left({ }^{H} I_{t_{0}}^{\alpha C H} D_{t_{0}}^{\alpha} x\right)(t)=x(t)-x\left(t_{0}\right)$. 


\section{A. Ardjouni and M. Haoues}

Definition 2.4. For any $x \in[a, b] \subset \mathbb{R}^{+}$, we define the upper-control function by

$$
U(t, x)=\sup \{f(t, s): a \leq s \leq x\}
$$

and the lower-control function by

$$
L(t, x)=\inf \{f(t, s): x \leq s \leq b\} .
$$

It is obvious that these functions are non-decreasing on $[a, b]$, i.e.

$$
L(t, x) \leq f(t, x) \leq U(t, x), t \in J
$$

Definition 2.5. A function $x \in X$ is positive bounded below if $x \in \mathcal{C}_{\theta}$. In particular, we call $x$ as nonnegative function if $p\left(t_{0}\right) \theta=0$ and positive function if $p\left(t_{0}\right) \theta>0$.

The following fixed point theorem due to Dhage [15] is essential tool for the proof of the first result.

Theorem 2.6 ([15]). Let $S$ be a nonempty bounded closed convex subset of a Banach algebra $X$. Let $\mathcal{B}: S \rightarrow X$ and $\mathcal{A}: S \rightarrow X$ be two operators such that

i) $\mathcal{A}$ is Lipschiz with a Lipschitz constant $\sigma$,

ii) $\mathcal{B}$ is completely continuous,

iii) $\mathcal{A} x \mathcal{B} y \in S$ for all $x, y \in S$.

Then the product operator equation

$$
\mathcal{A} x \mathcal{B} x=x,
$$

has a solution, whenever $\sigma M<1$, where $M=\sup \{\|\mathcal{B} x\|: x \in S\}$.

\section{Existence of positive solutions}

In this section, we will discuss the existence of positive solutions for (1.1). We introduce the following conditions

(H1) For $t \in J$ and $x \in X$, we have

$$
p(t)+\frac{1}{\Gamma(\beta)} \int_{t_{0}}^{t}\left(\log \frac{t}{s}\right)^{\beta-1} g(s, x(s)) \frac{d s}{s}>0
$$

and

$$
\theta+\frac{1}{\Gamma(\alpha)} \int_{t_{0}}^{t}\left(\log \frac{t}{s}\right)^{\alpha-1} f(s, x(s)) \frac{d s}{s} \geq 0 .
$$

(H2) Let $x^{*}, x_{*} \in \mathcal{C}_{\theta}$, such that $x_{*}\left(t_{0}\right)=x^{*}\left(t_{0}\right)=p\left(t_{0}\right) \theta$ and $p\left(t_{0}\right) \theta \leq x_{*}(t) \leq x^{*}(t) \leq b, t \in J$. Moreover,

$$
\left\{\begin{array}{c}
{ }^{C H} D_{t_{0}}^{\alpha}\left(\frac{x^{*}(t)}{p(t)+\frac{1}{\Gamma(\beta)} \int_{t_{0}}^{t}\left(\log \frac{t}{s}\right)^{\beta-1} g\left(s, x^{*}(s)\right) \frac{d s}{s}}\right) \geq U\left(t, x^{*}(t)\right), \\
{ }^{C H} D_{t_{0}}^{\alpha}\left(\frac{x_{*}(t)}{p(t)+\frac{1}{\Gamma(\beta)} \int_{t_{0}}^{t}\left(\log \frac{t}{s}\right)^{\beta-1} g\left(s, x_{*}(s)\right) \frac{d s}{s}}\right) \leq L\left(t, x_{*}(t)\right),
\end{array}\right.
$$

for any $t \in J$.

(H3) Let $g$ be monotonic non-decreasing with respect to $x$ and there exists $L_{g}>0$ such that

$$
|g(t, x)-g(t, y)| \leq L_{g}|x-y|, t \in J, x, y \in \mathbb{R}
$$


Hybrid Caputo-Hadamard fractional integro-differential equations

where

$$
0<L_{g} \frac{\left(\log \frac{T}{t_{0}}\right)^{\beta}}{\Gamma(\beta+1)}\left(|\theta|+\frac{c_{f}\left(\log \frac{T}{t_{0}}\right)^{\alpha}}{\Gamma(\alpha+1)}\right)<1
$$

(H4) There exists $L_{f}>0$ such that

$$
|f(t, x)-f(t, y)| \leq L_{f}|x-y|, t \in J, x, y \in \mathbb{R}
$$

The functions $x^{*}$ and $x_{*}$ are respectively called the pair of upper and lower solutions for (1.1).

From Lemma 2.3, we deduce the following lemma.

Lemma 3.1. Suppose that $\frac{x}{h}$ is differentiable on J. Then the equation

$$
\left\{\begin{array}{l}
{ }^{C H} D_{t_{0}}^{\alpha}\left(\frac{x(t)}{h(t)}\right)=f(t, x(t)), t \in J \\
x\left(t_{0}\right)=p\left(t_{0}\right) \theta
\end{array}\right.
$$

is equivalent to

$$
x(t)=h(t)\left(\theta+\frac{1}{\Gamma(\alpha)} \int_{t_{0}}^{t}\left(\log \frac{t}{s}\right)^{\alpha-1} f(s, x(s)) \frac{d s}{s}\right), t \in J
$$

By the previous lemma, (1.1) is equivalent to

$$
\begin{aligned}
x(t) & =\left(p(t)+\frac{1}{\Gamma(\beta)} \int_{t_{0}}^{t}\left(\log \frac{t}{s}\right)^{\beta-1} g(s, x(s)) \frac{d s}{s}\right) \\
& \times\left(\theta+\frac{1}{\Gamma(\alpha)} \int_{t_{0}}^{t}\left(\log \frac{t}{s}\right)^{\alpha-1} f(s, x(s)) \frac{d s}{s}\right), t \in J .
\end{aligned}
$$

Hence, according to the Dhage fixed point theorem 2.6, we define the operators $\mathcal{A}, \mathcal{B}: \mathcal{C}_{\theta} \rightarrow \mathcal{C}_{\theta}$ by

$$
(\mathcal{A} x)(t)=p(t)+\frac{1}{\Gamma(\beta)} \int_{t_{0}}^{t}\left(\log \frac{t}{s}\right)^{\beta-1} g(s, x(s)) \frac{d s}{s},
$$

and

$$
(\mathcal{B} x)(t)=\theta+\frac{1}{\Gamma(\alpha)} \int_{t_{0}}^{t}\left(\log \frac{t}{s}\right)^{\alpha-1} f(s, x(s)) \frac{d s}{s}
$$

for $t \in J$.

Theorem 3.2. Suppose that $(\mathbf{H 1})-(\mathbf{H 3})$ are satisfied, then the problem (1.1) has at last one positive bounded below solution $x \in \mathcal{C}_{\theta}$ satisfying $x_{*}(t) \leq x(t) \leq x^{*}(t), t \in J$.

Proof. Let $S=\left\{x \in \mathcal{C}_{\theta}: x_{*}(t) \leq x(t) \leq x^{*}(t), t \in J\right\}$, endowed with the norm $\|x\|=\max _{t \in J}|x(t)|$, then for any $x \in S$, we have $\|x\| \leq b$. Hence, $S$ is a convex, bounded and closed subset of $\mathcal{C}_{\theta}$. Moreover, the continuity of $g$ and $f$ implies the continuity of the operators $\mathcal{A}$ and $\mathcal{B}$ on $S$. Now, if $x \in S$ there exists a positive 


\section{A. Ardjouni and M. Haoues}

constant $c_{f}$ such that $\max \{|f(t, x(t))|:(t, x) \in J \times S\} \leq c_{f}$. Then

$$
\begin{aligned}
|(\mathcal{B} x)(t)| & =\left|\theta+\frac{1}{\Gamma(\alpha)} \int_{t_{0}}^{t}\left(\log \frac{t}{s}\right)^{\alpha-1} f(s, x(s)) \frac{d s}{s}\right| \\
& \leq|\theta|+\frac{1}{\Gamma(\alpha)} \int_{t_{0}}^{t}\left(\log \frac{t}{s}\right)^{\alpha-1}|f(s, x(s))| \frac{d s}{s} \\
& \leq|\theta|+\frac{c_{f}}{\Gamma(\alpha)} \int_{t_{0}}^{t}\left(\log \frac{t}{s}\right)^{\alpha-1} \frac{d s}{s} \\
& \leq|\theta|+\frac{c_{f}\left(\log \frac{t}{t_{0}}\right)^{\alpha}}{\Gamma(\alpha+1)}
\end{aligned}
$$

So,

$$
\|\mathcal{B} x\| \leq|\theta|+\frac{c_{f}\left(\log \frac{T}{t_{0}}\right)^{\alpha}}{\Gamma(\alpha+1)}
$$

Hence, $\mathcal{B}(S)$ is uniformly bounded. Next, we show the equicontinuity of $\mathcal{B}$. Let $x \in S$, then for any $t_{1}, t_{2} \in J$, $t_{2}>t_{1}$, we get

$$
\begin{aligned}
& \left|(\mathcal{B} x)\left(t_{2}\right)-(\mathcal{B} x)\left(t_{1}\right)\right| \\
& =\frac{1}{\Gamma(\alpha)}\left|\int_{t_{0}}^{t_{2}}\left(\log \frac{t_{2}}{s}\right)^{\alpha-1} f(s, x(s)) \frac{d s}{s}-\int_{t_{0}}^{t_{1}}\left(\log \frac{t_{1}}{s}\right)^{\alpha-1} f(s, x(s)) \frac{d s}{s}\right| \\
& \leq \frac{1}{\Gamma(\alpha)} \int_{t_{0}}^{t_{1}}\left(\left(\log \frac{t_{1}}{s}\right)^{\alpha-1}-\left(\log \frac{t_{2}}{s}\right)^{\alpha-1}\right)|f(s, x(s))| \frac{d s}{s} \\
& +\frac{1}{\Gamma(\alpha)} \int_{t_{1}}^{t_{2}}\left(\log \frac{t_{2}}{s}\right)^{\alpha-1}|f(s, x(s))| \frac{d s}{s} \\
& \leq \frac{c_{f}}{\Gamma(\alpha)}\left(\int_{t_{0}}^{t_{1}}\left(\log \frac{t_{1}}{s}\right)^{\alpha-1}-\left(\log \frac{t_{2}}{s}\right)^{\alpha-1} \frac{d s}{s}+\int_{t_{1}}^{t_{2}}\left(\log \frac{t_{2}}{s}\right)^{\alpha-1} \frac{d s}{s}\right) \\
& \leq \frac{c_{f}}{\Gamma(\alpha+1)}\left(\left(\log \frac{t_{1}}{t_{0}}\right)^{\alpha}-\left(\log \frac{t_{2}}{t_{0}}\right)^{\alpha}+2\left(\log \frac{t_{2}}{t_{1}}\right)^{\alpha}\right) \\
& \leq \frac{2 c_{f}}{\Gamma(\alpha+1)}\left(\log \frac{t_{2}}{t_{1}}\right)^{\alpha} \text {. }
\end{aligned}
$$

As $t_{1} \rightarrow t_{2}$ the right-hand side of the previous inequality is independent of $x$ and tends to zero. Therefore, $\mathcal{B}(S)$ is equicontinuous. The Arzela-Ascoli theorem implies that $\mathcal{B}$ is compact. Hence $\mathcal{B}$ is completely continuous. 
Hybrid Caputo-Hadamard fractional integro-differential equations

By hypothesis (H3), for any $x, y \in S$, we get

$$
\begin{aligned}
& |(\mathcal{A} x)(t)-(\mathcal{A} y)(t)| \\
& =\left|\frac{1}{\Gamma(\beta)} \int_{t_{0}}^{t}\left(\log \frac{t}{s}\right)^{\beta-1} g(s, x(s)) \frac{d s}{s}-\frac{1}{\Gamma(\beta)} \int_{t_{0}}^{t}\left(\log \frac{t}{s}\right)^{\beta-1} g(s, y(s)) \frac{d s}{s}\right| \\
& \leq \frac{1}{\Gamma(\beta)} \int_{t_{0}}^{t}\left(\log \frac{t}{s}\right)^{\beta-1}|g(s, x(s))-g(s, y(s))| \frac{d s}{s} \\
& \leq \frac{L_{g}}{\Gamma(\beta)}\left(\int_{t_{0}}^{t}\left(\log \frac{t}{s}\right)^{\beta-1} \frac{d s}{s}\right)\|x-y\| \\
& \leq \frac{L_{g}\left(\log \frac{T}{t_{0}}\right)^{\beta}}{\Gamma(\beta+1)}\|x-y\| .
\end{aligned}
$$

Then $\mathcal{A}$ is Lipschiz mapping with Lipschitz constant $\sigma=L_{g} \frac{\left(\log \frac{T}{t_{0}}\right)^{\beta}}{\Gamma(\beta+1)}$, that satisfying $\sigma \sup \{\|\mathcal{B} x\|: x \in S\}<$ 1.

We need to show that $\mathcal{A} x \mathcal{B} y \in S$ for all $x, y \in S$. Indeed, by Definition 2.4 and the hypothesis $(\mathbf{H 3})$, we obtain

$$
\begin{aligned}
& (\mathcal{A} x)(t)(\mathcal{B} y)(t) \\
& =\left(p(t)+\frac{1}{\Gamma(\beta)} \int_{t_{0}}^{t}\left(\log \frac{t}{s}\right)^{\beta-1} g(s, x(s)) \frac{d s}{s}\right)\left(\theta+\frac{1}{\Gamma(\alpha)} \int_{t_{0}}^{t}\left(\log \frac{t}{s}\right)^{\alpha-1} f(s, y(s)) \frac{d s}{s}\right) \\
& \leq\left(p(t)+\frac{1}{\Gamma(\beta)} \int_{t_{0}}^{t}\left(\log \frac{t}{s}\right)^{\beta-1} g(s, x(s)) \frac{d s}{s}\right)\left(\theta+\frac{1}{\Gamma(\alpha)} \int_{t_{0}}^{t}\left(\log \frac{t}{s}\right)^{\alpha-1} U(s, y(s)) \frac{d s}{s}\right) \\
& \leq\left(p(t)+\frac{1}{\Gamma(\beta)} \int_{t_{0}}^{t}\left(\log \frac{t}{s}\right)^{\beta-1} g\left(s, x^{*}(s)\right) \frac{d s}{s}\right)\left(\theta+\frac{1}{\Gamma(\alpha)} \int_{t_{0}}^{t}\left(\log \frac{t}{s}\right)^{\alpha-1} U\left(s, x^{*}(s)\right) \frac{d s}{s}\right) \\
& \leq x^{*}(t),
\end{aligned}
$$

and

$$
\begin{aligned}
& (\mathcal{A} x)(t)(\mathcal{B} y)(t) \\
& \geq\left(p(t)+\frac{1}{\Gamma(\beta)} \int_{t_{0}}^{t}\left(\log \frac{t}{s}\right)^{\beta-1} g(s, x(s)) \frac{d s}{s}\right)\left(\theta+\frac{1}{\Gamma(\alpha)} \int_{t_{0}}^{t}\left(\log \frac{t}{s}\right)^{\alpha-1} L(s, y(s)) \frac{d s}{s}\right) \\
& \geq\left(p(t)+\frac{1}{\Gamma(\beta)} \int_{t_{0}}^{t}\left(\log \frac{t}{s}\right)^{\beta-1} g\left(s, x_{*}(s)\right) \frac{d s}{s}\right)\left(\theta+\frac{1}{\Gamma(\alpha)} \int_{t_{0}}^{t}\left(\log \frac{t}{s}\right)^{\alpha-1} L\left(s, x_{*}(s)\right) \frac{d s}{s}\right) \\
& \geq x_{*}(t) .
\end{aligned}
$$

Hence, $x_{*}(t) \leq \mathcal{A} x(t) \mathcal{B} y(t) \leq x^{*}(t), t \in J$, that is $(\mathcal{A} x \mathcal{B} y)(S) \subseteq S$. According to the Dhage fixed point theorem, the operator equation $\mathcal{A} x \mathcal{B} x=x$ has at last one fixed point $x \in S$. Therefore, the problem (1.1) has at last one positive bounded below solution $x \in \mathcal{C}_{\theta}$.

Next, we consider many particular cases of the previous theorem.

Corollary 3.3. Suppose that (H3) holds and there exist $k_{1}, k_{2}, k_{3}, k_{4} \in X$, such that

$$
k_{1}(t) \leq g(t, x(t)) \leq k_{2}(t)
$$




\section{A. Ardjouni and M. Haoues}

and

$$
k_{3}(t) \leq f(t, x(t)) \leq k_{4}(t)
$$

If

$$
p(t)+\frac{1}{\Gamma(\beta)} \int_{t_{0}}^{t}\left(\log \frac{t}{s}\right)^{\beta-1} k_{1}(s) \frac{d s}{s}>0,
$$

and

$$
\theta+\frac{1}{\Gamma(\alpha)} \int_{t_{0}}^{t}\left(\log \frac{t}{s}\right)^{\alpha-1} k_{3}(s) \frac{d s}{s} \geq 0,
$$

then the problem (1.1) has at least one positive bounded below solution. Moreover

$$
\begin{aligned}
& \left(p(t)+\frac{1}{\Gamma(\beta)} \int_{t_{0}}^{t}\left(\log \frac{t}{s}\right)^{\beta-1} k_{1}(s) \frac{d s}{s}\right)\left(\theta+\frac{1}{\Gamma(\alpha)} \int_{t_{0}}^{t}\left(\log \frac{t}{s}\right)^{\alpha-1} k_{3}(s) \frac{d s}{s}\right) \\
& \leq x(t) \\
& \leq\left(p(t)+\frac{1}{\Gamma(\beta)} \int_{t_{0}}^{t}\left(\log \frac{t}{s}\right)^{\beta-1} k_{2}(s) \frac{d s}{s}\right)\left(\theta+\frac{1}{\Gamma(\alpha)} \int_{t_{0}}^{t}\left(\log \frac{t}{s}\right)^{\alpha-1} k_{4}(s) \frac{d s}{s}\right) .
\end{aligned}
$$

Proof. By the assumption (3.7) and the definition of control functions, we have

$$
k_{3}(t) \leq L(t, x(t)) \leq U(t, x(t)) \leq k_{4}(t),
$$

for any $t \in J$. Now, we consider the fractional differential equations

$$
{ }^{C H} D_{t_{0}}^{\alpha}\left(\frac{x(t)}{p(t)+\frac{1}{\Gamma(\beta)} \int_{t_{0}}^{t}\left(\log \frac{t}{s}\right)^{\beta-1} k_{1}(s) \frac{d s}{s}}\right)=k_{3}(t), x\left(t_{0}\right)=p\left(t_{0}\right) \theta,
$$

and

$$
{ }^{C H} D_{t_{0}}^{\alpha}\left(\frac{x(t)}{p(t)+\frac{1}{\Gamma(\beta)} \int_{t_{0}}^{t}\left(\log \frac{t}{s}\right)^{\beta-1} k_{2}(s) \frac{d s}{s}}\right)=k_{4}(t), x\left(t_{0}\right)=p\left(t_{0}\right) \theta .
$$

In accordance of Lemma 3.1, the solutions of (3.10) and (3.11) are given respectively by

$$
x(t)=\left(p(t)+\frac{1}{\Gamma(\beta)} \int_{t_{0}}^{t}\left(\log \frac{t}{s}\right)^{\beta-1} k_{1}(s) \frac{d s}{s}\right)\left(\theta+\frac{1}{\Gamma(\alpha)} \int_{t_{0}}^{t}\left(\log \frac{t}{s}\right)^{\alpha-1} k_{3}(s) \frac{d s}{s}\right),
$$

and

$$
x(t)=\left(p(t)+\frac{1}{\Gamma(\beta)} \int_{t_{0}}^{t}\left(\log \frac{t}{s}\right)^{\beta-1} k_{2}(s) \frac{d s}{s}\right)\left(\theta+\frac{1}{\Gamma(\alpha)} \int_{t_{0}}^{t}\left(\log \frac{t}{s}\right)^{\alpha-1} k_{4}(s) \frac{d s}{s}\right) .
$$

Therefore,

$$
\begin{aligned}
x(t) & =\left(p(t)+\frac{1}{\Gamma(\beta)} \int_{t_{0}}^{t}\left(\log \frac{t}{s}\right)^{\beta-1} k_{1}(s) \frac{d s}{s}\right)\left(\theta+\frac{1}{\Gamma(\alpha)} \int_{t_{0}}^{t}\left(\log \frac{t}{s}\right)^{\alpha-1} k_{3}(s) \frac{d s}{s}\right) \\
& \leq\left(p(t)+\frac{1}{\Gamma(\beta)} \int_{t_{0}}^{t}\left(\log \frac{t}{s}\right)^{\beta-1} k_{1}(s) \frac{d s}{s}\right)\left(\theta+\frac{1}{\Gamma(\alpha)} \int_{t_{0}}^{t}\left(\log \frac{t}{s}\right)^{\alpha-1} L(s, x(s)) \frac{d s}{s}\right),
\end{aligned}
$$


Hybrid Caputo-Hadamard fractional integro-differential equations

and

$$
\begin{aligned}
x(t) & =\left(p(t)+\frac{1}{\Gamma(\beta)} \int_{t_{0}}^{t}\left(\log \frac{t}{s}\right)^{\beta-1} k_{2}(s) \frac{d s}{s}\right)\left(\theta+\frac{1}{\Gamma(\alpha)} \int_{t_{0}}^{t}\left(\log \frac{t}{s}\right)^{\alpha-1} k_{4}(s) \frac{d s}{s}\right) \\
& \geq\left(p(t)+\frac{1}{\Gamma(\beta)} \int_{t_{0}}^{t}\left(\log \frac{t}{s}\right)^{\beta-1} k_{2}(s) \frac{d s}{s}\right)\left(\theta+\frac{1}{\Gamma(\alpha)} \int_{t_{0}}^{t}\left(\log \frac{t}{s}\right)^{\alpha-1} U(s, x(s)) \frac{d s}{s}\right)
\end{aligned}
$$

One can define the upper and lower solutions as

$$
x^{*}(t)=\left(p(t)+\frac{1}{\Gamma(\beta)} \int_{t_{0}}^{t}\left(\log \frac{t}{s}\right)^{\beta-1} k_{2}(s) \frac{d s}{s}\right)\left(\theta+\frac{1}{\Gamma(\alpha)} \int_{t_{0}}^{t}\left(\log \frac{t}{s}\right)^{\alpha-1} U\left(s, x^{*}(s)\right) \frac{d s}{s}\right),
$$

and

$$
x_{*}(t)=\left(p(t)+\frac{1}{\Gamma(\beta)} \int_{t_{0}}^{t}\left(\log \frac{t}{s}\right)^{\beta-1} k_{1}(s) \frac{d s}{s}\right)\left(\theta+\frac{1}{\Gamma(\alpha)} \int_{t_{0}}^{t}\left(\log \frac{t}{s}\right)^{\alpha-1} L\left(s, x_{*}(s)\right) \frac{d s}{s}\right) .
$$

Hence by Theorem 3.2, the problem (1.1) has a positive bounded below solution $x \in \mathcal{C}_{\theta}$.

Corollary 3.4. Let $k \in X$ and $\varphi \in \mathbb{R}_{+}$such that $\varphi<k(t)=\lim _{x \rightarrow \infty} f(t, x)<\infty$ for $t \in J$. If (H3), (3.6) and (3.8) hold and $\theta \in \mathbb{R}_{+}$, then the problem (1.1) has at least one positive bounded below solution. Moreover for $0<\omega<\varphi$,

$$
\begin{aligned}
& \left(p(t)+\frac{1}{\Gamma(\beta)} \int_{t_{0}}^{t}\left(\log \frac{t}{s}\right)^{\beta-1} k_{1}(s) \frac{d s}{s}\right)\left(\theta+\frac{(\varphi-\omega)\left(\log \frac{t}{t_{0}}\right)^{\alpha}}{\Gamma(\alpha+1)}\right) \\
& \leq x(t) \\
& \leq\left(p(t)+\frac{1}{\Gamma(\beta)} \int_{t_{0}}^{t}\left(\log \frac{t}{s}\right)^{\beta-1} k_{2}(s) \frac{d s}{s}\right)\left(\theta+\frac{1}{\Gamma(\alpha)} \int_{t_{0}}^{t}\left(\log \frac{t}{s}\right)^{\alpha-1} k(s) \frac{d s}{s}+\frac{\omega\left(\log \frac{t}{t_{0}}\right)^{\alpha}}{\Gamma(\alpha+1)}\right)
\end{aligned}
$$

Corollary 3.5. Suppose that (H3), (3.6) and (3.8) hold, and

$$
\lim _{x \rightarrow \theta} \frac{f(t, x)}{x}=\gamma(t)
$$

where $\gamma \in X, t \in J$. Then there exists a positive bounded below solution of the problem (1.1).

Corollary 3.6. Let $\mu, \nu$ and $\xi$ are real positive numbers such that $\mu \leq f(t, x(t)) \leq \nu x(t)+\xi$, for $t \in J$. If (3.6), (3.8) and (H3) hold and $\theta \in \mathbb{R}_{+}$, then the problem (1.1) has at least one positive bounded below solution. Moreover

$$
\begin{aligned}
& \left(p(t)+\frac{1}{\Gamma(\beta)} \int_{t_{0}}^{t}\left(\log \frac{t}{s}\right)^{\beta-1} k_{1}(s) \frac{d s}{s}\right)\left(\theta+\frac{\mu\left(\log \frac{t}{t_{0}}\right)^{\alpha}}{\Gamma(\alpha+1)}\right) \\
& \leq x(t) \\
& \leq\left(p(t)+\frac{1}{\Gamma(\beta)} \int_{t_{0}}^{t}\left(\log \frac{t}{s}\right)^{\beta-1} k_{2}(s) \frac{d s}{s}\right)\left(\theta+\frac{1}{\Gamma(\alpha)} \int_{t_{0}}^{t}\left(\log \frac{t}{s}\right)^{\alpha-1}(\nu x(s)+\xi) \frac{d s}{s}\right) .
\end{aligned}
$$




\section{A. Ardjouni and M. Haoues}

\section{Uniqueness of positive solutions}

In this portion, we will prove the uniqueness of the bounded below positive solution of (1.1) using the Banach contraction mapping principle.

Theorem 4.1. Suppose that $(\mathbf{H 1})-(\mathbf{H} 4)$ hold. If

$$
L_{g} \frac{\left(\log \frac{T}{t_{0}}\right)^{\beta}}{\Gamma(\beta+1)}\left(\theta+c_{f} \frac{\left(\log \frac{T}{t_{0}}\right)^{\alpha}}{\Gamma(\alpha+1)}\right)+\left(p_{m}+c_{g} \frac{\left(\log \frac{T}{t_{0}}\right)^{\beta}}{\Gamma(\beta+1)}\right) L_{f} \frac{\left(\log \frac{T}{t_{0}}\right)^{\alpha}}{\Gamma(\alpha+1)}<1
$$

then the problem (1.1) has a unique positive bounded below solution.

Proof. Let $c_{f}$ and $c_{g}$ are positive real numbers such that,

$$
|f(t, x(t))| \leq c_{f},|g(t, x(t))| \leq c_{g}
$$

for any $t \in J$ and $x, y \in \mathcal{C}_{\theta}$. According to Theorem 3.2, the problem (1.1) has at least one positive bounded below solution in $S$. Now, we need only to prove that the product operator $\mathcal{A} x \mathcal{B} x$ is a contraction mapping on $X$, where $\mathcal{A}$ and $\mathcal{B}$ are defined as in (3.4) and (3.5). Indeed, for any $x, y \in \mathcal{C}_{\theta}$ and $t \in J$, we get

$$
\begin{aligned}
& |(\mathcal{A} x \mathcal{B} x)(t)-(\mathcal{A} y \mathcal{B} y)(t)| \\
& \leq\left(\frac{1}{\Gamma(\beta)} \int_{t_{0}}^{t}\left(\log \frac{t}{s}\right)^{\beta-1}|g(s, x(s))-g(s, y(s))| \frac{d s}{s}\right)\left(\theta+\frac{1}{\Gamma(\alpha)} \int_{t_{0}}^{t}\left(\log \frac{t}{s}\right)^{\alpha-1}|f(s, x(s))| \frac{d s}{s}\right) \\
& +\left(|p(t)|+\frac{1}{\Gamma(\beta)} \int_{t_{0}}^{t}\left(\log \frac{t}{s}\right)^{\beta-1}|g(s, y(s))| \frac{d s}{s}\right) \\
& \times\left(\frac{1}{\Gamma(\alpha)} \int_{t_{0}}^{t}\left(\log \frac{t}{s}\right)^{\alpha-1}|f(s, x(x))-f(s, y(s))| \frac{d s}{s}\right) \\
& \leq\left(L_{g} \frac{\left(\log \frac{t}{t_{0}}\right)^{\beta}}{\Gamma(\beta+1)}\|x-y\|\right)\left(\theta+c_{f} \frac{\left(\log \frac{t}{t_{0}}\right)^{\alpha}}{\Gamma(\alpha+1)}\right) \\
& +\left(|p(t)|+c_{g} \frac{\left(\log \frac{t}{t_{0}}\right)^{\beta}}{\Gamma(\beta+1)}\right)\left(L_{f} \frac{\left(\log \frac{t}{t_{0}}\right)^{\alpha}}{\Gamma(\alpha+1)}\|x-y\|\right) \\
& \leq\left(L_{g} \frac{\left(\log \frac{T}{t_{0}}\right)^{\beta}}{\Gamma(\beta+1)}\left(\theta+c_{f} \frac{\left(\log \frac{T}{t_{0}}\right)^{\alpha}}{\Gamma(\alpha+1)}\right)+\left(p_{m}+c_{g} \frac{\left(\log \frac{T}{t_{0}}\right)^{\beta}}{\Gamma(\beta+1)}\right) L_{f} \frac{\left(\log \frac{T}{t_{0}}\right)^{\alpha}}{\Gamma(\alpha+1)}\right)\|x-y\|,
\end{aligned}
$$

where $p_{m}=\max _{t \in J}|p(t)|$. Hence, by (4.1) the product operator $\mathcal{A} x \mathcal{B} x$ is a contraction mapping. Therefore, the problem (1.1) has a unique positive bounded below solution $x \in \mathcal{C}_{\theta}$.

\section{Monotonicity of positive solutions}

Theorem 5.1. Let $p, g$ and $f$ be non-decreasing functions with respect to both variables, $f\left(t_{0}, x\left(t_{0}\right)\right) \geq 0$ and $g\left(t_{0}, x\left(t_{0}\right)\right) \geq 0$. Moreover, let $(\mathbf{H 1})-(\mathbf{H 4})$ hold, then there is a monotonic non-decreasing positive bounded below solution of the problem (1.1). 
Hybrid Caputo-Hadamard fractional integro-differential equations

Proof. Define a subset $R=\{x \in S: x$ is nondecreasing on $J\}$, then $R$ is a closed and convex subset of $S$. The operator $\mathcal{B}$ is uniformly bounded and completely continuous and the operator $\mathcal{A}$ is Lipschitzian with Lipschitz constant $\sigma$, and satisfying $\sigma \sup \{\|\mathcal{B} x\|: x \in R\} \leq 1$. It remains for applying the Dhage theorem that $\mathcal{A} x \mathcal{B} x \in$ $R$ whenever $x, y \in R$. To this end, it suffices to consider $x, y \in R, t_{1}, t_{2} \in J$ with $t_{1}<t_{2}$. It follows that

$$
\begin{aligned}
& \mathcal{A} x\left(t_{2}\right) \mathcal{B} y\left(t_{2}\right)-\mathcal{A} x\left(t_{1}\right) \mathcal{B} y\left(t_{1}\right) \\
& =\mathcal{A} x\left(t_{2}\right)\left(\mathcal{B} y\left(t_{2}\right)-\mathcal{B} y\left(t_{1}\right)\right)+\left(\mathcal{A} x\left(t_{2}\right)-\mathcal{A} x\left(t_{1}\right)\right) \mathcal{B} y\left(t_{1}\right) \\
& =\frac{1}{\Gamma(\alpha)}\left(p\left(t_{2}\right)+\frac{1}{\Gamma(\beta)} \int_{t_{0}}^{t_{2}}\left(\log \frac{t_{2}}{s}\right)^{\beta-1} g(s, x(s)) \frac{d s}{s}\right) \\
& \times\left(\int_{t_{0}}^{t_{1}}\left(\left(\log \frac{t_{2}}{s}\right)^{\alpha-1}-\left(\log \frac{t_{1}}{s}\right)^{\alpha-1}\right) f(s, y(s)) \frac{d s}{s}+\int_{t_{1}}^{t_{2}}\left(\log \frac{t_{2}}{s}\right)^{\alpha-1} f(s, y(s)) \frac{d s}{s}\right) \\
& +\left(p\left(t_{2}\right)-p\left(t_{1}\right)+\frac{1}{\Gamma(\beta)} \int_{t_{0}}^{t_{1}}\left(\left(\log \frac{t_{2}}{s}\right)^{\beta-1}-\left(\log \frac{t_{1}}{s}\right)^{\beta-1}\right) g(s, x(s)) \frac{d s}{s}\right. \\
& \left.+\frac{1}{\Gamma(\beta)} \int_{t_{1}}^{t_{2}}\left(\log \frac{t_{2}}{s}\right)^{\beta-1} g(s, x(s)) \frac{d s}{s}\right)\left(\theta+\frac{1}{\Gamma(\alpha)} \int_{t_{0}}^{t_{1}}\left(\log \frac{t_{1}}{s}\right)^{\alpha-1} f(s, y(s)) \frac{d s}{s}\right) .
\end{aligned}
$$

Since $\left(\log \frac{t_{2}}{s}\right)^{\alpha-1}-\left(\log \frac{t_{1}}{s}\right)^{\alpha-1}<0$ and $\left(\log \frac{t_{2}}{s}\right)^{\beta-1}-\left(\log \frac{t_{1}}{s}\right)^{\beta-1}<0$, then

$$
\begin{aligned}
& \mathcal{A} x\left(t_{2}\right) \mathcal{B} y\left(t_{2}\right)-\mathcal{A} x\left(t_{1}\right) \mathcal{B} y\left(t_{1}\right) \\
& \geq \frac{f\left(t_{1}, y\left(t_{1}\right)\right)}{\Gamma(\alpha)}\left(p\left(t_{2}\right)+\frac{1}{\Gamma(\beta)} \int_{t_{0}}^{t_{2}}\left(\log \frac{t_{2}}{s}\right)^{\beta-1} g(s, x(s)) \frac{d s}{s}\right) \\
& \times\left(\int_{t_{0}}^{t_{1}}\left(\left(\log \frac{t_{2}}{s}\right)^{\alpha-1}-\left(\log \frac{t_{1}}{s}\right)^{\alpha-1}\right) \frac{d s}{s}+\int_{t_{1}}^{t_{2}}\left(\log \frac{t_{2}}{s}\right)^{\alpha-1} \frac{d s}{s}\right) \\
& +\left(p\left(t_{2}\right)-p\left(t_{1}\right)+\frac{g\left(t_{1}, x\left(t_{1}\right)\right)}{\Gamma(\beta)}\left(\int_{t_{0}}^{t_{1}}\left(\left(\log \frac{t_{2}}{s}\right)^{\alpha-1}-\left(\log \frac{t_{1}}{s}\right)^{\alpha-1}\right) \frac{d s}{s}\right.\right. \\
& \left.\left.+\int_{t_{1}}^{t_{2}}\left(\log \frac{t_{2}}{s}\right)^{\alpha-1} \frac{d s}{s}\right)\right)\left(\theta+\frac{1}{\Gamma(\alpha)} \int_{t_{0}}^{t_{1}}\left(\log \frac{t_{1}}{s}\right)^{\alpha-1} f(s, y(s)) \frac{d s}{s}\right) \\
& \geq \frac{f\left(t_{1}, y\left(t_{1}\right)\right)}{\Gamma(\alpha+1)}\left(p\left(t_{2}\right)+\frac{1}{\Gamma(\beta)} \int_{t_{0}}^{t_{2}}\left(\log \frac{t_{2}}{s}\right)^{\beta-1} g(s, x(s)) \frac{d s}{s}\right)\left(\left(\log \frac{t_{2}}{t_{0}}\right)^{\alpha}-\left(\log \frac{t_{1}}{t_{0}}\right)^{\alpha}\right) \\
& +\left(p\left(t_{2}\right)-p\left(t_{1}\right)+\frac{g\left(t_{1}, x\left(t_{1}\right)\right)}{\Gamma(\beta+1)}\left(\left(\log \frac{t_{2}}{t_{0}}\right)^{\beta}-\left(\log \frac{t_{1}}{t_{0}}\right)^{\beta}\right)\right) \\
& \times\left(\theta+\frac{1}{\Gamma(\alpha)} \int_{t_{0}}^{t_{1}}\left(\log \frac{t_{1}}{s}\right)^{\alpha-1} f(s, y(s)) \frac{d s}{s}\right) \\
& \geq 0
\end{aligned}
$$

Therefore, with the Dhage fixed point theorem the product operator $\mathcal{A B}: R \rightarrow R$ has a fixed point with the positivity and monotonicity nondecreasing properties which is a solution of the problem (1.1).

Remark 5.2. The results of Theorem 5.1 are valid in Corollaries 3.3-3.6 if the assumptions of Theorem 5.1 are added to Corollaries 3.3-3.6. 


\section{A. Ardjouni and M. Haoues}

\section{Examples}

Example 6.1. Consider the Caputo-Hadamard fractional integro-differential equation

$$
\left\{\begin{array}{l}
{ }^{C} D^{\frac{2}{3}}\left(\frac{x(t)}{\frac{5+3 t}{8}+\frac{1}{\Gamma\left(\frac{1}{4}\right)} \int_{1}^{t}\left(\log \frac{t}{s}\right)^{-\frac{3}{4}}\left(\frac{x(s)+2}{x(s)+3}\right) \frac{d s}{s}}\right)=\frac{1}{5+t}\left(\frac{t x(t)}{x(t)+4}+5\right), t \in(1, e], \\
x(1)=0,
\end{array}\right.
$$

where $\alpha=2 / 3, \beta=1 / 4, \theta=0, p(t)=\frac{5+3 t}{8}, f(t, x)=\frac{1}{5+t}\left(\frac{t x}{x+4}+5\right)$ and $g(t, x)=\frac{x+2}{x+3}$. Since $g$ is nondecreasing on $x$,

$$
\frac{2}{3} \leq g(t, x) \leq 1 \text { and } \frac{5}{5+e} \leq f(t, x) \leq 1 \text { for } t \in[1, e], x \in[0,+\infty),
$$

and

$$
L_{g} \frac{\left(\log \frac{T}{t_{0}}\right)^{\beta}}{\Gamma(\beta+1)}\left(|\theta|+c_{f} \frac{\left(\log \frac{T}{t_{0}}\right)^{\alpha}}{\Gamma(\alpha+1)}\right) \simeq 0.136<1
$$

Then, by Corollary 3.3, (6.1) has a positive solution which verifies $x_{*}(t) \leq x(t) \leq x^{*}(t)$ where

$$
x_{*}(t)=\left(\frac{5+3 t}{8}+\frac{2(\log t)^{\frac{1}{4}}}{3 \Gamma\left(\frac{5}{4}\right)}\right) \frac{5}{5+e} \frac{(\log t)^{\frac{2}{3}}}{\Gamma\left(\frac{5}{3}\right)},
$$

and

$$
x^{*}(t)=\left(\frac{5+3 t}{8}+\frac{(\log t)^{\frac{1}{4}}}{\Gamma\left(\frac{5}{4}\right)}\right) \frac{(\log t)^{\frac{2}{3}}}{\Gamma\left(\frac{5}{3}\right)} .
$$

This positive solution is unique due to the condition (4.1) is satisfied since

$$
\begin{aligned}
& L_{g} \frac{\left(\log \frac{T}{t_{0}}\right)^{\beta}}{\Gamma(\beta+1)}\left(|\theta|+c_{f} \frac{\left(\log \frac{T}{t_{0}}\right)^{\alpha}}{\Gamma(\alpha+1)}\right)+\left(p_{m}+c_{g} \frac{\left(\log \frac{T}{t_{0}}\right)^{\beta}}{\Gamma(\beta+1)}\right) L_{f} \frac{\left(\log \frac{T}{t_{0}}\right)^{\alpha}}{\Gamma(\alpha+1)} \\
& \simeq 0.404<1 .
\end{aligned}
$$

The property of non-decreasing of this solution is not valid in spite of $f$.

Example 6.2. Consider the Caputo-Hadamard fractional integro-differential equation

$$
\left\{\begin{array}{l}
{ }^{C} D^{\frac{1}{3}}\left(\frac{x(t)}{\frac{t}{3}+1+\frac{1}{\Gamma\left(\frac{2}{5}\right)} \int_{1}^{t}\left(\log \frac{t}{s}\right)^{-\frac{3}{5}}(2-\cos (x(s))) \frac{d s}{s}}\right)=\frac{t}{6} \sin (x(t)), t \in(1, e], \\
x(1)=4
\end{array}\right.
$$

where $\alpha=1 / 3, \beta=2 / 5, \theta=1 / 3, p(t)=\frac{t}{3}+1, f(t, x)=\frac{t}{6} \sin x$ and $g(t, x)=2-\cos x$ for $t \in[1, e]$, $x \in\left[0, \frac{\pi}{2}\right]$. Hence, $g$ is nondecreasing on $x$ and

$$
0 \leq f(t, x) \leq \frac{e}{6}, 1 \leq g(t, x) \leq 2
$$


Hybrid Caputo-Hadamard fractional integro-differential equations

Since

$$
L_{g} \frac{\left(\log \frac{T}{t_{0}}\right)^{\beta}}{\Gamma(\beta+1)}\left(|\theta|+\frac{c_{f}\left(\log \frac{T}{t_{0}}\right)^{\alpha}}{\Gamma(\alpha+1)}\right) \simeq 0.947<1 .
$$

Then, by Corollary 3.3, (6.2) has a positive solution which verifies $x_{*}(t) \leq x(t) \leq x^{*}(t)$ where

$$
x_{*}(t)=\frac{t}{9}+\frac{1}{3}+\frac{(\log t)^{\frac{2}{5}}}{3 \Gamma\left(\frac{7}{5}\right)},
$$

and

$$
x^{*}(t)=\left(\frac{t}{3}+1+\frac{2(\log t)^{\frac{2}{5}}}{\Gamma\left(\frac{7}{5}\right)}\right)\left(\frac{1}{3}+\frac{e(\log t)^{\frac{1}{3}}}{6 \Gamma\left(\frac{4}{3}\right)}\right) .
$$

We could not guarantee this positive solution is unique due to the condition (4.1) is not satisfied. The property of non-decreasing of this positive solution is valid since $f$ and $g$ are increasing on $\left[0, \frac{\pi}{2}\right]$ and $p$ is increasing on $[1, e]$.

\section{Conclusion}

The hybrid nonlinear Caputo-Hadamard fractional integro-differential equation is considered. So, we have studied the existence, uniqueness and monotonicity of positive solutions. The main tool of this work is the method of upper and lower solutions and the Dhage and Banach fixed point theorems. However, by introducing a new fixed mapping, we obtain new positivity conditions.

\section{References}

[1] B. Ahmad AND S.K. NTouyas, Existence and uniqueness of solutions for Caputo-Hadamard sequential fractional order neutral functional differential equations, Electronic Journal of Differential Equations, 2017(36) (2017), 1-11.

[2] B. Ahmad and S.K. Ntouyas, Nonlocal boundary value problems for hybrid fractional differential equations and inclusions of Hadamard type, Fractional Differential Calculus, 5(2) (2015), 107-123.

[3] B. Ahmad AND S.K. NTouYas, Initial-value problems for hybrid Hadamard fractional differential equations, Electron. J. Differential Equations, 2014(161) (2014), 1-8.

[4] A. ARdjouni, Existence and uniqueness of positive solutions for nonlinear Caputo-Hadamard fractional differential equations, Proyecciones, 40(1) (2021), 139-152.

[5] A. ARdjouni, Positive solutions for nonlinear Hadamard fractional differential equations with integral boundary conditions, AIMS Mathematics, 4(4) (2019), 1101-1113.

[6] A. ARdjouni And A. Djoudi, Positive solutions for first-order nonlinear Caputo-Hadamard fractional relaxation differential equations, Kragujevac Journal of Mathematics, 45(6) (2021), 897-908.

[7] A. ARdjouni And A. Djoudi, Existence and uniqueness of positive solutions for first-order nonlinear Liouville-Caputo fractional differential equations, São Paulo J. Math. Sci., 14 (2020), 381-390.

[8] A. ARdjouni And A. Djoudi, Approximating solutions of nonlinear hybrid Caputo fractional integrodifferential equations via Dhage iteration principle, Ural Mathematical Journal, 5(1) (2019), 3-12. 


\section{A. Ardjouni and M. Haoues}

[9] A. ARdjouni AND A Djoudi, Existence and uniqueness of solutions for nonlinear hybrid implicit CaputoHadamard fractional differential equations, Results in Nonlinear Analysis, 2(3) (2019) 136-142.

[10] A. Ardjouni, A. Lachouri And A. Djoudi, Existence and uniqueness results for nonlinear hybrid implicit Caputo-Hadamard fractional differential equations, Open Journal of Mathematical Analysis, 3(2) (2019), $106-111$.

[11] H. Boulares, A. Ardjouni And Y. Laskri, Positive solutions for nonlinear fractional differential equations, Positivity, 21 (2017), 1201-1212.

[12] H. Boulares, A. Ardjouni And Y. Laskri, Stability in delay nonlinear fractional differential equations, Rend. Circ. Mat. Palermo, 65 (2016), 243-253.

[13] B.C. Dhage, Hybrid fixed point theory in partially ordered normed linear spaces and applications to fractional integral equations, Differ. Equ Appl., 5 (2013), 155-184.

[14] B.C. Dhage, G.T. Khurpe, A.Y. Shete And J.N. Salunke, Existence and approximate solutions for nonlinear hybrid fractional integro-differential equations, International Journal of Analysis and Applications, 11(2) (2016), 157-167.

[15] B.C. Dhage and V. Lakshmikantham, Basic results on hybrid differential equations, Nonlinear Anal. Hybrid Syst., 4 (2010), 414-424.

[16] F. GE AND C. Kou, Stability analysis by Krasnoselskii's fixed point theorem for nonlinear fractional differential equations, Applied Mathematics and Computation, 257 (2015), 308-316.

[17] F. GE AND C. Kou, Asymptotic stability of solutions of nonlinear fractional differential equations of order $1<\alpha<2$, Journal of Shanghai Normal University, 44(3) (2015), 284-290.

[18] M. Haoues, A. Ardjouni And A Djoudi, Existence, uniqueness and monotonicity of positive solutions for hybrid fractional integro-differential equations, Asia Mathematika, 4(3) (2020), 1-13

[19] A.A. Kilbas, H.M. Srivastava and J.J. Trujillo, Theory and Applications of Fractional Differential Equations, Elsevier Science B. V., Amsterdam, 2006.

[20] N. Li AND C. WANG, New existence results of positive solution for a class of nonlinear fractional differential equations, Acta Math. Sci., 33 (2013) 847-854.

[21] M. MATAR, Qualitative properties of solution for hybrid nonlinear fractional differential equations, Afrika Matematika, 30(7-8) (2019), 1169-1179.

[22] Y. Zhao, S. Sun, Z. Han And Q. Li, Theory of fractional hybrid differential equations, Comput. Math. Appl., 62 (2011), 1312-1324.

This is an open access article distributed under the Creative Commons Attribution License, which permits unrestricted use, distribution, and reproduction in any medium, provided the original work is properly cited. 